\title{
COVID-19 pandemic: a double trouble for Indian adolescents and young adults living with type 1 diabetes
}

\author{
Neha Agarwal $^{1} \cdot$ Mandara Harikar $^{2}\left(\right.$ [D $\cdot$ Rishi Shukla ${ }^{1,3} \cdot$ Anurag Bajpai ${ }^{1,3}$ \\ Received: 20 July 2020 / Accepted: 24 August 2020 / Published online: 18 September 2020 \\ (C) Research Society for Study of Diabetes in India 2020
}

\begin{abstract}
Background Strict isolation measures and interrupted health care services during the COVID 19 pandemic are contemplated to instigate stress universally, particularly in those with chronic illnesses such as type 1 diabetes (T1D).

Methods A cross-sectional, observational study was done to assess determinants of stress and its impact on glycemic control in adolescents and young adults (aged 12-24 years) living with T1D in India. An online, semi-structured survey including Perceived Stress Scale (PSS-10) was distributed and results were analyzed.

Results A total of 89 participants (46 males, mean age $19.61 \pm 3.8$ years) with T1D completed the survey. Age $(r=0.325, p=$ $0.005)$ and HBA1C level within the preceding 3 months $(r=0.274, p=0.036)$ correlated positively with PSS-10 scores. There was a statistically significant difference in PSS-10 score based on gender $(t(70)=-2.147 ; p=0.035)$, education $(F(4,67)=4.34$, $p=0.003)$, and occupation $(F(3,68)=4.50, p=.006)$. On multiple linear regression, gender, occupation, and HbA1C were the significant determinants of PSS-10 $\left(F(3,55)=12.01, p<0.001, R^{2}=0.363\right)$. One-way ANOVA showed a significant impact of mean PSS-10 score on the glycemic control $(F(2,69)=3.813, p=0.027)$.

Conclusion An increased prevalence of stress was seen among Indian adolescents and young adults living with T1D. Female gender, salaried individuals, and pre-existing poorly controlled diabetes contributed to an increased risk of stress. Increased stress resulted in worsened glycemic control.
\end{abstract}

Keywords COVID-19 $\cdot$ PSS-10 · Type 1 diabetes $\cdot$ Adolescent

\section{Introduction}

Coronavirus disease (COVID-19), caused by a novel coronavirus SARS-CoV-2, is considered a close relative of severe acute respiratory syndrome (SARS) [1]. With the first COVID-19 case detected in December 2019 in Wuhan, Hubei province of China [2], it has spread rampantly, jeopardizing health infrastructure and economies across the globe. COVID-19 was officially declared a pandemic by the World Health Organization (WHO) on 11th March 2020 [3].

Mandara Harikar

GROW Society, Growth and Obesity Workforce, Kanpur, India

Independent researcher, Rishikesh, India

3 Department of Endocrinology, Regency Healthcare, Kanpur, India
In the absence of an effective drug or vaccine against COVID-19, prevention is by far the best way to limit the infection [4]. In an attempt to mitigate the spread of the disease, an unprecedented practice of social distancing has been instituted across the nation [5]. Strict isolation measures, interruption in access to routine health care, compromised academic, and social activities are contemplated to instigate stress and anxiety among all, particularly those living with chronic illnesses, such as type 1 diabetes (T1D) [6].

T1D is one of the most common endocrine metabolic disorders around the world [7]. Individuals living with T1D are at an increased risk of psychological issues, owing either to the underlying disease, or due to the complexity involved in the management of diabetes [8]. Diabetes management mandates adherence to insulin, balanced diet, regular physical activity, and self-monitoring of blood glucose in order to achieve good glycemic control and prevent the development of short-term and long-term complications [9]. 
Studies have reported the psychological impact of the COVID-19 pandemic on health care workers, children, and older adults [10-12]. However, there is scarcity of data regarding the psychological impact of COVID-19 on those living with chronic illnesses, such as T1D.

We believe that this is the first study to analyze the level of perceived stress among Indian adolescents and young adults living with T1D, using a validated psychometric tool (Perceived Stress Scale, PSS-10), and identify the determinants of stress and its impact on the glycemic control.

\section{Methodology}

This was a cross-sectional, observational study, involving known patients living with T1D. An online survey was designed using Google Forms and shared with the participants. The online link comprised of an informed consent followed by a semi-structured questionnaire. Participants were included if they were between 12 and 24 years, living with T1D, had an ability to understand simple English, had access to the internet, and were willing to participate in the study. Data collection was started on 30th April 2020 at 4 PM IST and closed on 10th May 2020 at 4 PM IST. After accepting to participate in the survey, participants were redirected to the four different sections of the questionnaire (Appendix I).

The first section included demographic details; the second included assessment of knowledge and attitude of participants towards COVID-19 (based on the information and recommendations provided by the World Health Organization [13] and the Ministry of Health and Family Welfare, India) [14]. Each question was awarded 1 point for the correct response and 0 for an incorrect response. The scores for transmission, prevention, and presentation were converted to percentages of total possible score. The third section evaluated the presence of stress among the participants using the ten-item Perceived Stress Scale (PSS-10) [15] and the techniques adopted to cope with stress. PSS-10 measures the psychological stress estimated over the previous 4 weeks. It consists of 10 items measured on a five-point Likert scale ( 0 never, 1 almost never, 2 sometimes, 3 fairly often, 4 very often). The total score is obtained by adding the scores of all the items, with reverse coding for items $4,5,7$, and 8 , as they are positively stated. The total score ranges from 0 to 40 , with score 40 depicting the highest perceived stress level. Participants were categorized into "low stress" for PSS-10 score between 0 and 13, "moderate stress" for scores 14-26, and "high stress" if PSS-10 score between 27 and 40 [15].

The fourth section included questions pertaining to T1D, HBA1C within the preceding 3 months, change in frequency of self-monitoring of blood glucose (SMBG), self-assessed glycemic control based on SMBG, possible reasons for poor blood sugar control, and potential ways of allaying stress.

\section{Statistical analysis}

The data was compiled using Microsoft Excel and analyzed using IBM Statistical Package for Social Sciences (SPSS version 25.0, SPSS, Inc., Chicago, IL, USA). Continuous variables were expressed as mean (SD) and categorical variables as frequencies (percentages). Student's $t$ test for independent samples and oneway ANOVA were used to compare the means of PSS-10 score among different groups of participants. Spearman correlation was used to test for correlation between non-parametric, continuous variables. Variables found to have a significant association with PSS-10 score on univariate analysis were entered as predictor variables into a multiple linear regression model, with PSS-10 score as the dependent variable. A $p$ value of less than 0.05 was considered as significant.

\section{Results}

A total of 89 adolescents and young adults (46 males, mean age $19.61 \pm 3.8$ years), living with type 1 diabetes, with a mean duration of diabetes $8.4 \pm 5.0$ years and a mean HBA1C of $8.1 \pm 1.5 \%$, completed the survey. Majority were on basal bolus regime ( $80.9 \%$ ) followed by split mix regime $(13.5 \%)$; and a small proportion of them were on continuous subcutaneous insulin infusion (CSII; 5.6\%). The baseline socio-demographic characteristics of the participants is depicted in Table 1.

All the 89 participants had either heard or read about the COVID-19 pandemic. The prime source of information was television $(74.2 \%)$ followed by social media $(67.4 \%)$, search engines (64\%), print media (59.6\%), and lastly governmentauthorized applications $(53.9 \%)$. Around $78.7 \%(n=70)$ of the participants were reportedly satisfied with the available information, with almost all $(89.9 \%, n=80)$ tracking pandemic-related information at least once daily. Table 2 details the performance of participants on questions regarding the transmission, prevention, and presentation of COVID-19 infection.

More than half of all the cases $51.7 \%(n=46)$ reported moderate stress; low stress was perceived by $42.7 \%(n=38)$ and severe stress was observed in 5.6\% $(n=5)$. Age correlated positively with mean PSS-10 score $(r=0.325, p=0.005)$. Females were found to have a significantly higher PSS-10 score compared with males $(19.03 \pm 5.39$ versus $15.97 \pm$ $6.61 ; t(70)=-2.147 p=0.035)$. There was a statistically significant difference in PSS-10 score between respondents, based on their education $(F(4,67)=4.34, p=.003)$ and occupation $(F(3,68)=4.50, p=0.006)$. A Tukey post hoc test showed that those in high school had significantly lesser PSS-10 score compared with the graduates $(p=0.020)$ and post-graduates $(p=0.002)$. Furthermore, Tukey post hoc test revealed that those employed had significantly higher PSS-10 score compared with students $(p=0.020)$. 
Table 1 Baseline socio-demographic characteristics of the study participants

\begin{tabular}{lll}
\hline Variables & Type & Participants $n(\%)$ \\
\hline Age (year) & & $19.61 \pm 3.82^{*}$ \\
Gender & Male & $46(51.7)$ \\
& Female & $43(48.3)$ \\
Marital Status & Married & $2(2.2)$ \\
& Unmarried & $87(97.8)$ \\
Residence & Independent house & $67(75.3)$ \\
& Apartment & $20(22.5)$ \\
& Hostel & $2(2.2)$ \\
Education & Less than high school & $15(16.8)$ \\
& High school & $8(9.0)$ \\
& Intermediate & $13(14.6)$ \\
& Graduation & $41(46.1)$ \\
& Post-graduation & $12(13.5)$ \\
& Student & $63(70.8)$ \\
Occupation & Employed & $13(14.6)$ \\
& Business & $7(7.9)$ \\
& Unemployed & $6(6.7)$ \\
\hline
\end{tabular}

${ }^{*}$ Mean $\pm \mathrm{SD}$

Participants who were reportedly dissatisfied with the pandemic-related information were found to have significantly higher PSS-10 score $(F(2,69)=3.440, p=0.038)$. A significant positive correlation was obtained between the mean PSS-10 score and HBA1C level within the preceding 3 months $(r=0.274, p=0.036$, Fig. 1). No significant association with PSS-10 score was observed for the type of residence, knowledge level regarding transmission, prevention, and presentation of COVID-19 infection, the frequency of seeking COVID-19-related information, presence of any suspected symptom over the last 14 days, duration of diabetes, and insulin regimen (Table 3 ).

On multiple linear regression analysis, age, gender, education, occupation, satisfaction with available information, and $\mathrm{HbA} 1 \mathrm{C}$ were entered as predictor variables. On stepwise backward linear regression analysis, the model comprising gender, occupation, and HbA1C on PSS-10 score was significant with $F(3,55)=12.01, p<0.001, R^{2}=0.363$.

Reduced frequency of SMBG was reported by $55.1 \%$ ( $n=$ 49) of the participants. Based on self-assessment of SMBG over the last 1 month, improved glycemic control was reported by $42.7 \%$ ( $n=38)$, worsened glycemic control by $13.5 \%$ $(n=12)$, and $43.8 \%(n=39)$ reported no change. One-way ANOVA showed a significant impact of mean PSS-10 score on the glycemic control $(F(2,69)=3.813, p=0.027)$. Selfreported worsening of glycemic control was significantly more common among those with a higher mean PSS-10 score compared with those who reported no change $(p=0.021)$. Table 4 enumerates the challenges faced by the participants in diabetes management and their potential solutions.

Among the various coping methods reported by the participants, spending time with friends and family was reported by the majority $(78.6 \%)$, followed by pursuit of hobbies $(61.1 \%)$ and praying $(50 \%)$.
Table 2 Summary of correct responses for COVID-19-related information

\begin{tabular}{ll}
\hline COVID-19 transmission, prevention, and presentation & Participants $n(\%)$ \\
\hline How does the COVID-19 infection spread? & $79(88.8)$ \\
Direct contact with infected person & $72(80.9)$ \\
Droplet & $22(24.7)$ \\
Airborne & \\
Do not know & $3(3.4)$ \\
How can you protect yourself from the COVID-19 infection? & \\
Frequent hand washing & $84(94.4)$ \\
Social distancing & $84(94.4)$ \\
Wearing a face-mask when outdoors & $85(95.5)$ \\
Avoiding contact with persons coughing/sneezing & $81(91.0)$ \\
Do not know & 0 \\
What are the presentation of COVID-19 infection? & $83(93.3)$ \\
Fever & $82(92.1)$ \\
Dry cough & $56(62.9)$ \\
Tiredness & $57(64.0)$ \\
Cold & $24(27.0)$ \\
Diarrhea & $57(64)$ \\
Sometimes, no symptoms & $3(3.4)$ \\
Do not know & \\
\# The infection remains in the air for long periods of time, even when people have moved away.
\end{tabular}


Table 3 Determinants of Perceived Stress Scale (PSS-10) among study participants

\begin{tabular}{|c|c|c|c|}
\hline Demographic & Type & PSS-10 score $($ mean \pm SD) & $p$ value* \\
\hline Age (year) & & $r=0.325^{\#}$ & $0.005^{*}$ \\
\hline Gender & $\begin{array}{l}\text { Male } \\
\text { Female }\end{array}$ & $\begin{array}{l}15.97 \pm 6.61 \\
19.03 \pm 5.39\end{array}$ & $0.035^{*}$ \\
\hline Residence & $\begin{array}{l}\text { Independent house } \\
\text { Apartment } \\
\text { Hostel }\end{array}$ & $\begin{array}{l}16.58 \pm 6.08 \\
20.29 \pm 6.18 \\
18 \pm 2.82\end{array}$ & 0.097 \\
\hline Education & $\begin{array}{l}\text { Less than high school } \\
\text { High school } \\
\text { Intermediate } \\
\text { Graduate } \\
\text { Post-graduate }\end{array}$ & $\begin{array}{l}13 \pm 6.52 \\
17.8 \pm 7.79 \\
16.5 \pm 6.11 \\
18.52 \pm 5.14 \\
22.62 \pm 3.96\end{array}$ & $0.003 *$ \\
\hline Occupation & $\begin{array}{l}\text { Student } \\
\text { Employed } \\
\text { Business } \\
\text { Unemployed }\end{array}$ & $\begin{array}{l}16.32 \pm 6.19 \\
22.3 \pm 3.36 \\
23.25 \pm 5.56 \\
15.83 \pm 4.79\end{array}$ & $0.006^{*}$ \\
\hline Presence of any symptom over last 14 days & $\begin{array}{l}\text { Yes } \\
\text { No }\end{array}$ & $\begin{array}{l}19.11 \pm 5.11 \\
17.26 \pm 6.33\end{array}$ & 0.4 \\
\hline Satisfaction with available information & $\begin{array}{l}\text { Yes } \\
\text { No } \\
\text { Do not know }\end{array}$ & $\begin{array}{l}16.59 \pm 6.33 \\
22.67 \pm 5.08 \\
19.5 \pm 3.89\end{array}$ & $0.038 *$ \\
\hline Frequency of accessing information & $\begin{array}{l}\text { Never } \\
\text { Occasionally } \\
\text { Daily } \\
\text { Multiple times a day }\end{array}$ & $\begin{array}{l}23 \pm 4.24 \\
21 \pm 3.69 \\
16 \pm 6.24 \\
18.56 \pm 6.16\end{array}$ & 0.074 \\
\hline Knowledge of COVID-19 infection & $\begin{array}{l}\text { Transmission score } \\
\text { Prevention Score } \\
\text { Symptom Score }\end{array}$ & $\begin{array}{l}r=-0.149^{\#} \\
r=-0.089^{\#} \\
r=-0.085^{\#}\end{array}$ & $\begin{array}{l}0.194 \\
0.438 \\
0.462\end{array}$ \\
\hline Duration of type 1 diabetes (year) & & $r=-0.042^{\#}$ & 0.717 \\
\hline Insulin regimen & $\begin{array}{l}\text { Split mix regimen } \\
\text { Basal bolus } \\
\text { Continuous infusion }\end{array}$ & $\begin{array}{l}19.70 \pm 5.47 \\
17.04 \pm 6.13 \\
18.40 \pm 8.29\end{array}$ & 0.435 \\
\hline
\end{tabular}

${ }^{*} p$ value $<0.05$ is considered significant

${ }^{\text {\# }}$ Spearman correlation
Fig. 1 Spearman correlation showing positive association of PSS-10 with $\mathrm{HbA1C}$

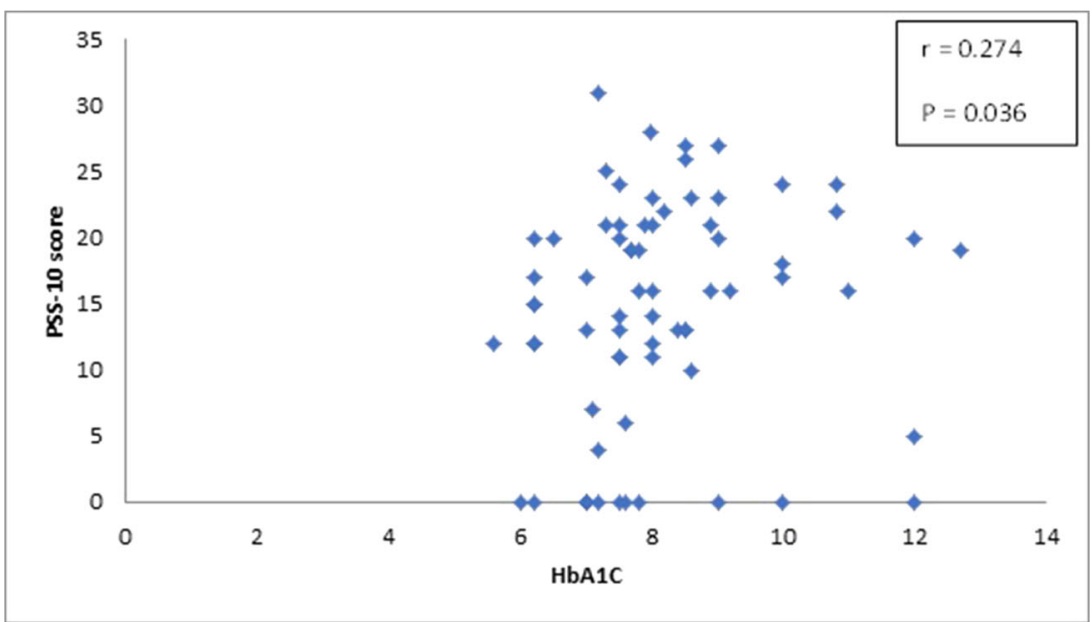


Table 4 Challenges faced in diabetes management and potential solutions

\begin{tabular}{ll}
\hline & Participants $n(\%)$ \\
\hline Reason for poor sugar control & \\
Difficulty in getting consultations & $12(9.6)$ \\
Difficulty in managing healthy diet & $41(33.06)$ \\
Lack of physical work & $40(32.25)$ \\
Unavailability of blood sugar monitoring strips & $22(17.74)$ \\
Unavailability of insulin & $9(7.25)$ \\
Potential solutions for better management & \\
Information on T1D care during COVID & $46(51.7)$ \\
Online consultations & $58(65.2)$ \\
Easy availability of insulin and glucometer strips & $60(67.4)$ \\
\hline
\end{tabular}

\section{Discussion}

The COVID-19 pandemic has emerged as a double trouble for those living with T1D. In an attempt to curb the spread of COVID-19, the Government of India announced a nationwide lockdown on 24th March 2020 by the PM [5]. This much needed step taken towards the containment of the virus has, to some extent, adversely affected the availability of routine health care facilities, supply of drugs, and medical equipment [6]. The limitation in access to health care facilities, drugs, and other resources, along with the known vulnerability to infection due to a compromised immune system, has left many with apprehension and increased stress levels [16].

In the present study, which was conducted just 5 weeks after the announcement of the nationwide lockdown, around $94.4 \%$ of the study participants reported low to moderate stress. The soaring stress level observed within the initial few weeks of the lockdown is an alarming finding in our study. Similar to the finding reported by Gao et al. [17], stress correlated positively with the age in our study. A better understanding of the impact of the pandemic, and professional uncertainty for an age group that either is planning or has recently started their career, could be a plausible explanation for this positive association. In agreement to previous epidemiological studies, which reported females to be at a higher risk for psychological disorders, we too noted that perceived stress was more among females compared with males [18, 19]. Similar to the findings reported by Du et al. [20], those with higher education were observed to have greater stress compared with their counterparts. This could possibly be attributed to their older age, or a result of apprehensions stemming from increased awareness regarding the pandemic, or both. In contradiction to increased stress reported among students by Wang et al. [19], we observed that salaried individuals perceived significantly more stress compared with students, selfemployed or unemployed individuals. The uncertainty and potential negative impact on professional life could possibly explain the increased perception of stress among the salaried individuals.

All infectious outbreaks have their own unique set of characteristics in terms of mode of spread, type of illness caused, and preventive measures to be adopted. The fear of getting infected or infecting others is a common occurrence during the infectious pandemics. Provision of accurate health-related information to the masses is a crucial step in mitigating this fear and stress. A significantly higher PSS-10 score was seen among those who were reportedly dissatisfied with the available information. In contradiction to past studies [17], no difference in perceived stress was observed with the source of information. This could be because of smaller sample size in our study.

We observed a significantly higher PSS-10 score among those with poorly controlled diabetes, as reflected by the reported HBA1C within the preceding 3 months. This could be attributed to the fact that the current pandemic, possibly, augmented their pre-existing health-related stress. Previous reports have described the negative impact of stress on the medication adherence and disease outcome [21]. A similar trend was found in our study, where reduced frequency of SMBG was reported by the participants.

Stress and glycemic control have a bidirectional relationship. It can directly lead to disturbance in glucose regulation [22], or can indirectly lead to non-adherence to medication and healthy lifestyles [23]. We found that participants with a higher PSS-10 score reported worsened glycemic control on SMBG in the preceding 4 weeks. This is in contrast to other studies performed in developed countries that demonstrated no deterioration [24, 25], or even an improvement [26] in glycemic control during the lockdown period, attributed to improved self-care. This dissimilarity could possibly be attributed to the differences in the study population. Considering that India is an emerging economy, there is an expected difference in the availability of medical supplies and financial stability between patients.

Knowledge regarding preventive measures among the study group was remarkably good. This is in contrast to previous studies that have reported inadequate levels of knowledge about the measures of prevention towards the pandemic among the general public [27]. The positive trend in our study could be attributed to the vigorous measures taken by the government and the media to reinforce healthy practices and minimize misinformation. Also, most respondents in our study possessed at least high school level education and also suffered from an underlying chronic illness, thus likely to be more sensitized to such information. Unlike other studies [19, 20], we did not find any significant association of PSS-10 score with the level of knowledge towards COVID-19 transmission, prevention, and presentation.

Our study showed that majority of the participants obtained their information primarily through television and social media 
sources. This is in agreement to previous studies which reported internet and television as prime sources of information during infectious outbreaks $[28,29]$. As evident from our study, adolescents and young adults are observed to be more inclined towards the digital platforms; health authorities could utilize this resource to spread knowledge and awareness regarding the pandemic and also to provide psychological support to those at an increased risk of mental health-related issues.

People adopted different strategies to cope with stress during this outbreak. Sharing problems with friends and family members was the most common strategy adopted. This is similar to the findings reported in previous studies where participants reported increased need to talk with someone to vent out their distress [30].

Restricted mobility during the lockdown has compromised the care of T1D patients, due to disruption in follow-up visits, restricted availability of medicines, and other equipment. Majority of the study participants expressed the need for easy availability of insulin and glucometer strips as an indispensable measure to allay their stress. Telephonic or online consultations along with the provision of specific protocols for diabetes management during the COVID-19 were also suggested by around two-thirds of the respondents.

\section{Limitations}

Restriction of the study design to those with access to smartphones and English proficiency limits the extrapolation of results across all the sections of the society. Also, there could have been a response bias as the participants might have opted for the most socially acceptable responses. Furthermore, we used PSS-10, a subjective tool to assess mental health, and the results of this may not tally with the objective assessment tools. Similarly, in the absence of an objective assessment of physical activity and diet, impact of these two variables could not be analyzed on the glycemic control. But, given the current scenario of lockdown, remote collection of data using a self-reported online questionnaire was the most apt way to conduct this study. Glycemic control was assessed with SMBG, owing to the limitations in $\mathrm{HbA1C}$ testing during the times of the pandemic. Notwithstanding the above limitations, this study provides an invaluable information about the challenges faced by the individuals living with T1D, amidst the current pandemic. It provides a reference for further studies in this area. It highlights the need to formulate strategies to mitigate stress among those at risk, particularly when the difficulties are bound to increase due to the ongoing pandemic.

To conclude, we found that an increased prevalence of stress was seen among the Indian adolescents and young adults living with T1D. Female gender, salaried individuals, and pre-existing poorly controlled diabetes contributed to an increased risk of stress. Increased stress resulted in worsened glycemic control on SMBG. Amidst the focus of containing and defeating the disease, health care facilities might miss out on those with chronic illnesses, whose management may worsen during the pandemic.

Acknowledgements The authors would like to thank the study participants for generously taking their time out to participate in the survey.

\section{Compliance with ethical standards}

Conflict of interest The authors declare that they have no conflict of interest.

Research involving human participants Yes.

Ethics committee approval Ethical clearance obtained.

Informed consent Obtained from every study participant prior to their participation in the survey.

\section{References}

1. Paules CI, Marston HD, Fauci AS. Coronavirus infections-more than just the common cold. JAMA. 2020;323:707-8.

2. Nishiura H, Jung SM, Linton NM, Kinoshita R, Yang Y, Hayashi $\mathrm{K}$, et al. The extent of transmission of novel coronavirus in Wuhan, China, 2020. J Clin Med. 2020 Jan 24;9(2):330. https://doi.org/10. 3390/jcm9020330.

3. Cucinotta D, Vanelli M. WHO declares COVID-19 a pandemic. Acta Biomed. 2020;91:157-60.

4. Bavel JJV, Baicker K, Boggio PS, Capraro V, Cichocka A, Cikara $\mathrm{M}$, et al. Using social and behavioural science to support COVID19 pandemic response. Nat Hum Behav. 2020 May;4(5):460-71. https://doi.org/10.1038/s41562-020-0884-z.

5. Lancet T. India under COVID-19 lockdown. Lancet. 2020;395: 1315.

6. Kretchy IA, Asiedu-Danso M, Kretchy JP. Medication management and adherence during the COVID-19 pandemic: Perspectives and experiences from low-and middle-income countries. Res Social Adm Pharm. 2020 Apr 15;S1551-7411(20): 30332-6. https://doi.org/10.1016/j.sapharm.2020.04.007.

7. Tuomilehto J. The emerging global epidemic of type 1 diabetes. Curr Diab Rep. 2013;13(6):795-804.

8. Kalra S, Jena BN, Yeravdekar R. Emotional and psychological needs of people with diabetes. Indian J Endocrinol Metab. 2018;22:696.

9. Donaghue KC, Marcovecchio ML, Wadwa RP, Chew EY, Wong TY, Calliari LE, et al. ISPAD clinical practice consensus guidelines 2018: microvascular and macrovascular complications in children and adolescents. Pediatr Diabetes. 2018;19(Suppl 27):262-74.

10. Lai J, Ma S, Wang Y, Cai Z, Hu J, Wei N, et al. Factors associated with mental health outcomes among health care workers exposed to coronavirus disease 2019. JAMA Netw Open. 2020 Mar 2;3(3): e203976. https://doi.org/10.1001/jamanetworkopen.2020.3976.

11. Golberstein E, Wen H, Miller BF. Coronavirus Disease 2019 (COVID-19) and mental health for children and adolescents. JAMA Pediatr. 2020, Apr 14. https://doi.org/10.1001/ jamapediatrics.2020.1456.

12. Banerjee D. The impact of Covid-19 pandemic on elderly mental health. Int J Geriatr Psychiatry. 2020 May 4:10.1002/gps.5320. https://doi.org/10.1002/gps.5320. 
13. Advice for the public on COVID-19 - World Health Organization [Internet]. Who.int. 2020 [cited 24 April 2020]. Available from: https://www.who.int/emergencies/diseases/novel-coronavirus2019/advice-for-public.

14. MoHFW | Home [Internet]. Mohfw.gov.in. 2020 [cited 24 April 2020]. Available from: https://mohfw.gov.in/.

15. Cohen S, Kamarck T, Mermelstein R. A global measure of perceived stress. J Health Soc Behav. 1983;24:386-96.

16. Huang Y, Zhao N. Generalized anxiety disorder, depressive symptoms and sleep quality during COVID-19 outbreak in China: a webbased cross-sectional survey. Psychiatry Res. 2020;12:112954.

17. Gao J, Zheng P, Jia Y, Chen H, Mao Y, Chen S, et al. Mental health problems and social media exposure during COVID-19 outbreak. PLoS One. 2020;15(4):e0231924.

18. Özdin S, Bayrak Özdin Ș. Levels and predictors of anxiety, depression and health anxiety during COVID-19 pandemic in Turkish society: the importance of gender. Int J Soc Psychiatry. 2020;66: 504-11.

19. Wang C, Pan R, Wan X, et al. Immediate psychological responses and associated factors during the initial stage of the 2019 coronavirus disease (COVID-19) epidemic among the general population in China. Int J Environ Res Public Health. 2020;17(5):1729 Published 2020. https://doi.org/10.3390/ijerph17051729.

20. Du J, Dong L, Wang T, et al. Psychological symptoms among frontline healthcare workers during COVID-19 outbreak in Wuhan [published online ahead of print, 2020 Apr 3]. Gen Hosp Psychiatry. 2020;S0163-8343(20):30045-1.

21. Cohen DM, Lumley MA, Naar-King S, Partridge T, Cakan N. Child behavior problems and family functioning as predictors of adherence and glycemic control in economically disadvantaged children with type 1 diabetes: a prospective study. J Pediatr Psychol. 2004;29(3):171-84.

22. Landsberg L, Young JB. Sympathoadrenal system. In: Contemporary endocrinology. Boston: Springer; 1985. p. 217-46.

23. Horii T, Momo K, Yasu T, Kabeya Y, Atsuda K. Determination of factors affecting medication adherence in type 2 diabetes mellitus patients using a nationwide claim-based database in Japan. PLoS One. 2019 Oct 8;14(10):e0223431. https://doi.org/10.1371/journal. pone. 0223431 .

24. Tornese G, Ceconi V, Monasta L, Carletti C, Faleschini E, Barbi E. Diabetes Technology \& Therapeutics. Jun 2020:462-7. https://doi. org/10.1089/dia.2020.0169.
25. Beato-Víbora PI. RETRACTED: No deleterious effect of lockdown due to COVID-19 pandemic on glycaemic control, measured by glucose monitoring, in adults with type 1 diabetes. Diabetes Technol Ther. 2020 May 12. https://doi.org/10.1089/dia.2020. 0184. Retraction in: Diabetes Technol Ther. 2020 Aug;22(8):643.

26. Bonora BM, Boscari F, Avogaro A, Bruttomesso D, Fadini GP. Glycaemic control among people with type 1 diabetes during lockdown for the SARS-CoV-2 outbreak in Italy. Diabetes Ther. 2020 May 11;11(6):1-11. https://doi.org/10.1007/s13300-020-00829-7.

27. Johnson EJ, Hariharan S. Public health awareness: knowledge, attitude and behaviour of the general public on health risks during the H1N1 influenza pandemic. J Public Health. 2017;25(3):333-7.

28. Chandrasekaran N, Gressick K, Singh V, et al. The utility of social media in providing information on zika virus. Cureus. 2017 Oct;9(10):e1792. https://doi.org/10.7759/cureus.1792.

29. Fung IC, Duke CH, Finch KC, Snook KR, Tseng PL, Hernandez $\mathrm{AC}$, et al. Ebola virus disease and social media: a systematic review. Am J Infect Control. 2016;44(12):1660-71.

30. Lee AA, Piette JD, Heisler M, Rosland AM. Diabetes distress and glycemic control: the buffering effect of autonomy support from important family members and friends. Diabetes Care. 2018;41(6):1157-63.

What is already known?

1. Infectious outbreaks are known to impact mental health adversely.

2. Those with chronic illnesses, like type 1 diabetes, are more vulnerable to psychological stress.

What this study adds?

1. Female gender, salaried individuals, and pre-existing poorly controlled diabetes contributed to an increased risk of stress.

2. Increased stress resulted in worsened glycemic control on SMBG.

Publisher's note Springer Nature remains neutral with regard to jurisdictional claims in published maps and institutional affiliations. 